Aleix Prat, Joel S. Parker, and Charles M. Perou, University of North Carolina, Chapel Hill, NC; Aleix Prat, Vall d'Hebron Institute of Oncology and Universitat Autònoma de Barcelona, Barcelona; Miguel Martín, Instituto de Investigacion Sanitaria Hospital Universitario Gregorio Marañón, Facultad de Medicina, Universidad Complutense; Miguel Martín, Eva Carrasco, and Rosalía Caballero, Grupo Español de Investigación en Cáncer de Mama, Madrid, Spain; Maggie Chon U. Cheang, Scott Tyldesley, Karen Gelmon, and Torsten O. Nielsen, British Columbia Cancer Agency; Maggie Chon U. Cheang, Scott Tyldesley, Karen Gelmon, and Torsten O. Nielsen, University of British Columbia, Vancouver, British Columbia, Canada; and Philip S. Bernard, University of Utah Health Sciences Center, Salt Lake City, UT.

Published online ahead of print at www.jco.org on December 10, 2012

Supported by funds from the $\mathrm{NCl}$ Breast SPORE program (Grant No. P50-CA58223-09A1), by Grant No. RO1-CA138255, by the Breast Cancer Research Foundation, and by the Sociedad Española de Oncología Médica. A.P. is affiliated with the Medicine $\mathrm{PhD}$ program of the Autonomous University of Barcelona, Spain

Presented in part at the IMPAKT Breast Cancer Conference, 3-5 May, 2012.

Brussels, Belgium

Authors' disclosures of potential conflicts of interest and author contributions are

found at the end of this article

Corresponding author: Aleix Prat, MD, Vall d'Hebron Institute of Oncology (VHIO), Pg Vall d'Hebron, 119-129, 08035, Barcelona, Spain; e-mail: aprat@vhio.net.

๑ 2012 by American Society of Clinical Oncology

0732-183X/13/3102-203/\$20.00

DOI: 10.1200/JCO.2012.43.4134

\title{
Prognostic Significance of Progesterone Receptor-Positive Tumor Cells Within Immunohistochemically Defined Luminal A Breast Cancer
}

Aleix Prat, Maggie Chon U. Cheang, Miguel Martín, Joel S. Parker, Eva Carrasco, Rosalía Caballero, Scott Tyldesley, Karen Gelmon, Philip S. Bernard, Torsten O. Nielsen, and Charles M. Perou

\section{$\begin{array}{lllllllll}\text { A } & \text { B } & \text { S } & \text { T } & \text { R } & \text { A } & \text { C } & \text { T }\end{array}$}

\section{Purpose}

Current immunohistochemical ( $\mathrm{HHC}$-based definitions of luminal A and B breast cancers are imperfect when compared with multigene expression-based assays. In this study, we sought to improve the IHC subtyping by examining the pathologic and gene expression characteristics of genomically defined luminal A and B subtypes.

\section{Patients and Methods}

Gene expression and pathologic features were collected from primary tumors across five independent cohorts: British Columbia Cancer Agency (BCCA) tamoxifen-treated only, Grupo Español de Investigación en Cáncer de Mama 9906 trial, BCCA no systemic treatment cohort, PAM50 microarray training data set, and a combined publicly available microarray data set. Optimal cutoffs of percentage of progesterone receptor (PR) -positive tumor cells to predict survival were derived and independently tested. Multivariable Cox models were used to test the prognostic significance.

\section{Results}

Clinicopathologic comparisons among luminal A and B subtypes consistently identified higher rates of PR positivity, human epidermal growth factor receptor 2 (HER2) negativity, and histologic grade 1 in luminal A tumors. Quantitative PR gene and protein expression were also found to be significantly higher in luminal A tumors. An empiric cutoff of more than $20 \%$ of PR-positive tumor cells was statistically chosen and proved significant for predicting survival differences within IHC-defined luminal A tumors independently of endocrine therapy administration. Finally, no additional prognostic value within hormonal receptor $(H R)$-positive/HER2negative disease was observed with the use of the IHC4 score when intrinsic IHC-based subtypes were used that included the more than $20 \%$ PR-positive tumor cells and vice versa.

\section{Conclusion}

Semiquantitative IHC expression of PR adds prognostic value within the current IHC-based luminal A definition by improving the identification of good outcome breast cancers. The new proposed IHC-based definition of luminal A tumors is HR positive/HER2 negative/Ki-67 less than 14\%, and PR more than $20 \%$.

\section{J Clin Oncol 31:203-209. (C) 2012 by American Society of Clinical Oncology}

\section{INTRODUCTION}

Hormonal receptor (HR) -positive breast cancer is a clinically and biologically heterogeneous entity. ${ }^{1-3}$ Studies based on gene expression profiling have identified at least two major groups of HR-positive tumors, known as the luminal A and $B$ intrinsic subtypes of breast cancer. These two molecular entities have shown significant differences in baseline prognosis and sensitivity to cytotoxic therapies. $^{4-6}$

Currently, a gene expression-based assay known as the PAM50 subtype predictor identifies the intrinsic molecular subtypes of breast cancer and provides a risk of relapse (ROR) score in a fashion similar to the Oncotype DX (Genomic Health, Redwood City, CA) recurrence score (RS). ${ }^{4-6}$ These two assays provide valuable and independent prognostic information beyond standard clinicopathologic variables. However, standardized gene expressionbased tests are not readily available in most of the world as a result of cost, assay turnaround times, and other logistic issues. Thus surrogate definitions of the intrinsic subtypes and/or risk of relapse groups developed using routine pathology and clinical parameters could be of great practical value. ${ }^{7,8}$ 
We have previously reported an immunohistochemical (IHC)based surrogate definition of the luminal A (IHC-luminal A) and luminal B/human epidermal growth factor receptor 2 (HER2) -negative (IHC-luminal B/HER2-negative) subtypes based on the quantitative expression of the proliferation-related marker Ki-67 within HR-positive/HER2-negative disease. ${ }^{9}$ This definition has now been adopted by the 2011 St Gallen Expert Consensus Panel Recommendation Guidelines for the systemic treatment of early breast cancer, ${ }^{10}$ which recommend adjuvant endocrine therapy alone for patients with IHC-luminal A tumors and the addition of chemotherapy for patients with IHC-luminal B/HER2-negative tumors. Here we further refine the IHC-based definition of luminal A and B through the use of quantitative progesterone receptor (PR) expression.

\section{PATIENTS AND METHODS}

\section{Patients, Samples, and Clinical Data}

Multiple different and independent data sets were used to assess the significance of PR IHC results. Gene expression and/or clinicopathologic features were evaluated across five different data sets: (1) a combined genomic data set of nine publicly available microarray cohorts (GSE18229, GSE18864, GSE22219, GSE25066, GSE2990, GSE4922, GSE7390, GSE7849, and NKI295), (2) the PAM50 microarray-based subtype predictor training data set (PAM50-training, GSE10886), ${ }^{5}$ (3) a British Columbia Cancer Agency (BCCA) tamoxifen-treated cohort (BCCA-tamoxifen), ${ }^{6}(4)$ the Grupo Español de Investigación en Cáncer de Mama (GEICAM) 9906 trial, ${ }^{11}$ and (5) the BCCA no adjuvant systemic therapy (AST) cohort (BCCA-no AST). ${ }^{9}$ A detailed CONSORT diagram can be found in Appendix Table A1 (online only).

All patients from the BCCA-tamoxifen cohort ${ }^{6}$ had early-stage HRpositive disease and received adjuvant treatment with tamoxifen only. In the GEICAM 9906 phase III trial cohort, ${ }^{11}$ patients with node-positive disease were randomly assigned to adjuvant fluorouracil, epirubicin, and cyclophosphamide versus fluorouracil, epirubicin, and cyclophosphamide followed by weekly paclitaxel, and patients with HR-positive disease subsequently received adjuvant endocrine therapy. The BCCA-no AST cohort" includes "clinically low risk" patients with primary breast cancer diagnosed between 1986 and 1992 who did not receive adjuvant systemic therapy. Characteristics of both BCCA cohorts and the GEICAM 9906 cohort have been previously described. ${ }^{6,9,11}$ From the PAM50-training cohort, we performed global and single gene expression analyses using only the prototypical samples of the luminal A and B subtype. Finally, the combined microarray data set included nine publicly available data sets of primary breast cancers with annotated clinicopathologic data.

\section{PAM50 Intrinsic Subtyping}

All tumors were assigned an intrinsic molecular subtypes of breast cancer (luminal A, luminal B, HER2-enriched, and basal-like) and the normal-like group using the PAM50 subtype predictor. ${ }^{5,6}$ In the BCCA-tamoxifen and GEICAM 9906 cohorts, ${ }^{11}$ PAM50 was determined using a quantitative reverse-transcriptase polymerase chain reaction-based assay., ${ }^{5,6}$ In the GEICAM 9906 cohort, we evaluated the PAM50 ROR score based on subtype and proliferation (ROR-P) as previously described for the BCCA-tamoxifen cohort. ${ }^{6}$ In each individual publicly available microarray cohort, we applied the PAM50 microarray-based algorithm ${ }^{5}$ after data set to data set normalization based on median gene centering within each data set.

IHC-based subtyping was determined using the following definitions adopted by the 2011 St Gallen Consensus Panel ${ }^{10}$ : IHC-luminal A (HR positive/HER2 negative/Ki-67 < 14\%), IHC-luminal B/HER2-negative (HR positive/HER2 negative/Ki-67 > 14\%), IHC-luminal B/HER2-positive (HR positive/HER2 positive), IHC-HER2 + (HR negative/HER2 positive), and triple-negative (HR negative/HER2 negative). Detailed IHC-based protocols for estrogen receptor (ER), PR, HER2, and Ki-67 determinations have been previously described ${ }^{6,9,11,12}$ and are summarized in Appendix Table A2 (on- line only). All IHC-based tissue microarray images of both BCCA cohorts can be obtained via the Genetic Pathology Evaluation Centre TMA Viewer. ${ }^{13}$

\section{IHC4 Score}

A version of the IHC4 score was evaluated in HER2-negative disease using the reported formula. ${ }^{8}$ However, instead of using the $\mathrm{H}$-score reported in Cuzick et $\mathrm{al}^{8}$ for estimating the semiquantitative expression of ER, we determined a general intensity score value of 0 to 3 and multiplied this value by the percentage of ER-positive tumor cells for a final ER score of 0 to 300 .

\section{Statistical Analysis}

Significant differences in clinicopathologic features between groups were evaluated using either the $\chi^{2}$ test or the $t$ test. Estimates of survival were from the Kaplan-Meier curves and tests of differences by the log-rank test. Univariate and multivariate Cox models were used to test the independent prognostic significance of each variable. Over-represented biologic processes were identified with Expression Analysis Systematic Explorer (EASE). ${ }^{14}$

To identify an optimal cutoff of percentage of PR-positive tumor cells within IHC-luminal A tumors, we applied the penalized spline method on multivariable Cox regression analysis in the BCCA-tamoxifen cohort $^{6}$ (training data set), and the optimal cutoff to predict distant relapse-free survival (DRFS) was independently tested in the GEICAM $9906^{11}$ and BCCA-no AST $^{9}$ cohorts.

To test the contribution of the IHC4 score, IHC-based subtyping and the PAM50 ROR-P score, all of these variables were tested in a prognostic model within HR-positive/HER2-negative disease. Here we estimated the log likelihood ratio statistic of each variable as an addition to a model containing the following clinical variables in the GEICAM 9906 cohort $^{11}$ : treatment arm, histologic grade, tumor stage, nodal status, and age. Finally, we estimated the $\log$ likelihood ratio statistic of each variable as an addition to a model containing clinical variables and one or two of the three variables being evaluated (IHC4 score, intrinsic IHC-based subtyping, and PAM50 ROR-P).

\section{RESULTS}

\section{Gene and Protein Expression Differences Between Luminal $A$ and $B$ Tumors}

To identify global and single gene expression differences, we performed a two-class significance analysis of microarrays between prototypical luminal A and B tumors from the PAM50-training cohort. ${ }^{5}$ A total of 1,539 genes (348 upregulated and 1,191 downregulated) were found differentially expressed (false discovery rate $<1 \%$ ) between both subtypes (Appendix Fig A1, online only; Data Supplement). The upregulated gene list in luminal A tumors was found enriched for genes involved in cell differentiation (eg, Kruppel-like factor 4 and jun proto-oncogene) and cell adhesion (eg, vinculin and collagen, type XVI, $\alpha 1$ ) biologic processes. Conversely, the downregulated gene list in luminal A tumors (ie, genes highly expressed in luminal B tumors) was found enriched for genes involved in immune response (eg, interleukin 2 receptor $\alpha$ and CD86) and cell-cycle (eg, cyclin $\mathrm{B} 1$ and RAD51) biologic processes, which is indicative of the faster proliferation rates known to be part of luminal B tumors.

Among the relatively upregulated genes in luminal A tumors was the progesterone receptor gene $(P G R)$, but not the estrogen receptor gene (ESR1). To further explore these findings, we evaluated the mRNA expression of PGR and ESR1 in two independent studies in which PAM50 was performed using the quantitative reversetranscriptase polymerase chain reaction platform (GEICAM $9906^{11}$ and BCCA-tamoxifen ${ }^{6}$ ) and confirmed that $P G R$, but not ESR1, was found significantly upregulated in luminal A tumors compared with luminal $\mathrm{B}$ tumors (Figs $1 \mathrm{~A}$ and $1 \mathrm{~B} ; P<.001, t$ test). Interestingly, $P G R$ was found only weakly correlated (Pearson correlation coefficient $=$ 

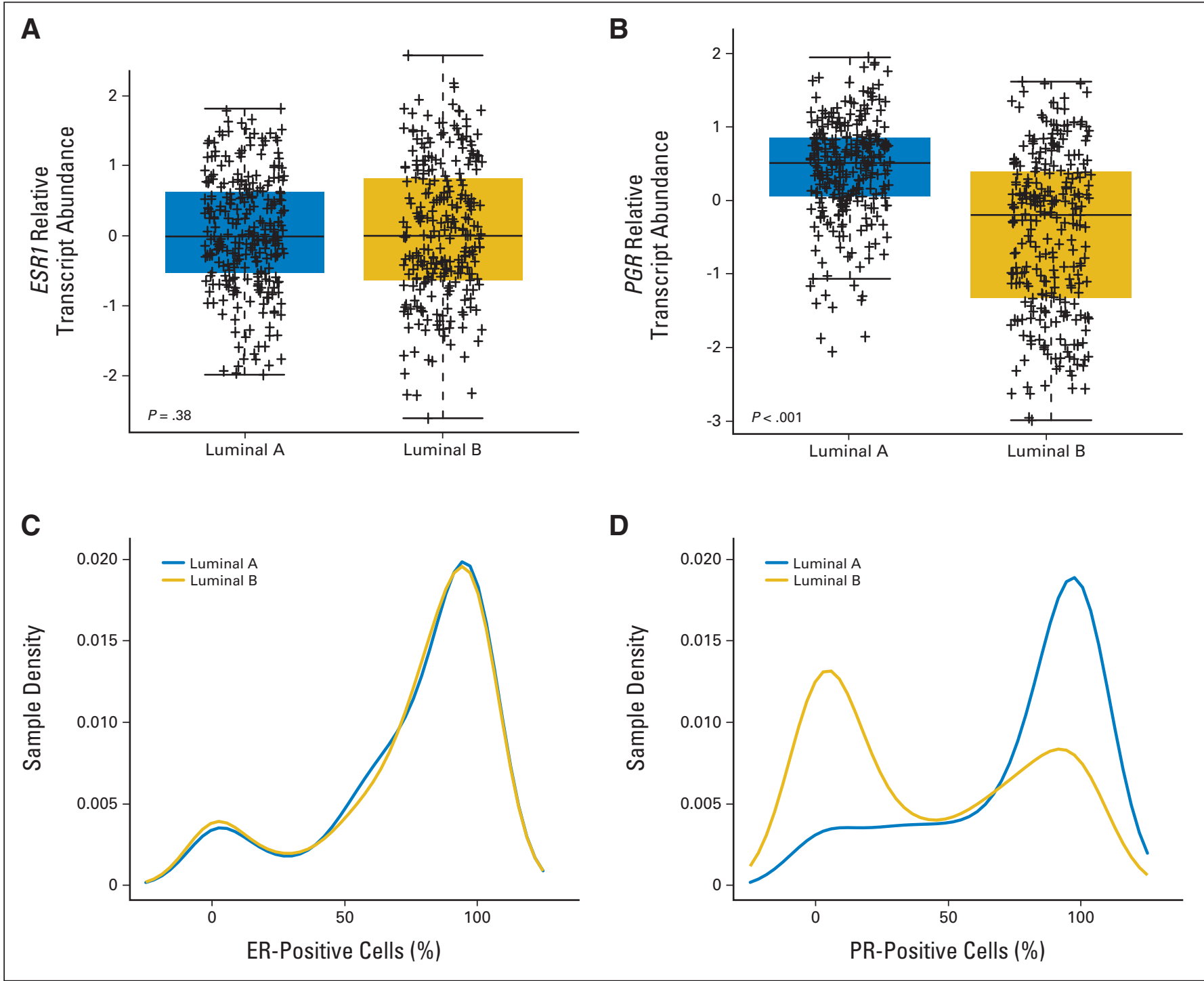

Fig 1. Expression of the hormonal receptors in the Grupo Español de Investigación en Cáncer de Mama 9906 data set. (A) Estrogen receptor (ER) gene (ESR1) and (B) progesterone receptor (PR) gene (PGR) as assayed using quantitative reverse-transcriptase polymerase chain reaction expression in luminal A and B tumors. Density plots based on the percentage of (C) ER-positive and (D) PR-positive tumor cells as assessed by immunohistochemistry.

-0.19) with the expression of the Ki-67 gene MKI67, indicating that these two genes may provide different biologic information.

The mRNA expression-based data suggested that semiquantitative scoring of the PR protein, but not ER protein, might help discriminate the genomically defined luminal A from B tumors. To further explore this hypothesis, we compared the percentage of PR-positive and ER-positive tumor cells as assessed by IHC, among luminal A and B tumors in the GEICAM 9906 cohort, ${ }^{11}$ and observed that only the percentage of PR-positive cells can discriminate luminal A from B tumors (Figs 1C and 1D). However, it is important to note that considerable overlap was observed. Finally, PR protein expression was also weakly anticorrelated with Ki-67 protein expression $(r=-0.20)$.

\section{Clinicopathologic Features of Luminal $A$ and $B$ Tumors}

To identify clinicopathologic differences among the genomically defined luminal A and B tumors, we evaluated the clinico- pathologic features of 2,257 patients with luminal A or B primary breast cancer. Across three independent cohorts (Table 1), luminal A tumors showed significantly higher rates of PR positivity, HER2 negativity, histologic grade 1, and tumor stage T0-T1 compared with luminal B tumors. No significant differences in ER status were observed, with the vast majority (92\% to $96 \%$ ) of luminal A and B tumors being ER positive.

\section{IHC-Based Versus PAM50 Subtype Definitions}

Current IHC-based definitions of luminal A and B subtypes are imperfect when compared with multigene expression-based assays. ${ }^{5}$ To further illustrate this, we evaluated the distribution of the IHC-based definitions within luminal A and B tumors in the BCCAtamoxifen $^{6}$ and the GEICAM 9906 cohorts. $^{11}$ As expected, whereas a large majority ( $81 \%$ to $85 \%$ ) of luminal A tumors were identified as IHC-luminal A, 35\% to $52 \%$ of luminal B tumors were also identified as IHC-luminal A (Table 2). 


\begin{tabular}{|c|c|c|c|c|c|c|c|c|c|c|c|c|c|c|c|}
\hline \multirow[b]{3}{*}{ Variable } & \multicolumn{5}{|c|}{$\begin{array}{l}\text { BCCA-Tamoxifen } \\
\text { ER-Positive Only }\end{array}$} & \multicolumn{5}{|c|}{$\begin{array}{l}\text { GEICAM } 9906 \\
\text { Node Positive }\end{array}$} & \multicolumn{5}{|c|}{$\begin{array}{l}\text { Combined Microarray Dataset } \\
\text { All }\end{array}$} \\
\hline & \multicolumn{2}{|c|}{ Luminal A } & \multicolumn{2}{|c|}{ Luminal B } & \multirow[b]{2}{*}{$P$} & \multicolumn{2}{|c|}{ Luminal A } & \multicolumn{2}{|c|}{ Luminal B } & \multirow[b]{2}{*}{$P$} & \multicolumn{2}{|c|}{ Luminal A } & \multicolumn{2}{|c|}{ Luminal B } & \multirow[b]{2}{*}{$P$} \\
\hline & No. & $\%$ & No. & $\%$ & & No. & $\%$ & No. & $\%$ & & No. & $\%$ & No. & $\%$ & \\
\hline No. & 372 & & 329 & & - & 278 & & 264 & & - & 594 & & 414 & & - \\
\hline Mean age, years & 66.6 & & 67.4 & & $>.05$ & 50.8 & & 51.7 & & $>.05$ & 53.5 & & 55.2 & & .03 \\
\hline \multicolumn{16}{|l|}{ Grade } \\
\hline 1 & 25 & 7 & 5 & 2 & $<.001$ & 69 & 25 & 26 & 10 & $<.001$ & 173 & 82 & 38 & 18 & $<.001$ \\
\hline 2 & 186 & 54 & 129 & 41 & & 141 & 51 & 112 & 42 & & 272 & 64 & 152 & 36 & \\
\hline 3 & 135 & 39 & 179 & 57 & & 68 & 10 & 126 & 47 & & 96 & 35 & 176 & 65 & \\
\hline Nodal positivity & 245 & 72.1 & 215 & 69 & $>.05$ & - & & - & & - & 220 & 38 & 195 & 49 & .002 \\
\hline Tumor size $>2.0 \mathrm{~cm}$ & 150 & 44 & 165 & 56 & .003 & 136 & 49 & 166 & 63 & .0031 & 341 & 57 & 280 & 68 & .001 \\
\hline IHC ER-positive status & - & & - & & - & 257 & 93 & 240 & 92 & $>.05$ & 552 & 94 & 390 & 95 & .583 \\
\hline IHC PR-positive status & 248 & 72 & 174 & 56 & $<.001$ & 261 & 94 & 195 & 74 & $<.001$ & 206 & 80 & 99 & 66 & .001 \\
\hline Clinical HER2-positive status & 15 & 4 & 30 & 9 & .0067 & 4 & 2 & 37 & 14 & $<.001$ & 14 & 6 & 19 & 14 & .008 \\
\hline
\end{tabular}

Finally, we explored the survival of the luminal A and B subtypes within the IHC-based luminal A and IHC-luminal B/HER2-negative tumors in the BCCA-tamoxifen cohort ${ }^{6}$ (Appendix Table A3, online only). In both cases, luminal A tumors showed a significantly better DRFS outcome than non-luminal A tumors. In multivariable Cox model survival analyses adjusted for histologic grade, age at diagnosis, nodal positivity, and tumor size, the hazard ratio for DRFS in PAM50 luminal A tumors compared with PAM50 non-luminal A was 0.642 within IHC-luminal A tumors ( $95 \% \mathrm{CI}, 0.422$ to $0.975, P=.038$ ) and 0.582 within IHC-luminal B/HER2-negative tumors ( $95 \%$ CI, 0.323 to $1.047, P=.071)$.

\section{Survival Outcomes Based on the Percentage of PR-Positive Cells}

These data suggested that (1) further improvements in the IHCluminal A definition is needed because many PAM50-defined luminal B tumors are erroneously identified as IHC-luminal A and (2) quantitative scoring of PR-positive tumor cells, but not ER-positive tumor cells, might help identify good-outcome breast cancers. To test this hypothesis, we evaluated the association of the visually determined percentage of PR-positive and ER-positive invasive breast carcinoma cells with survival outcomes within IHC-luminal A tumors of the BCCAtamoxifen cohort. ${ }^{6}$ As expected, the percentage of PR-positive cancer cells, but not the percentage of ER-positive cancer cells (data not shown), was associated with DRFS after adjusting for standard clinicopathologic variables, with the optimal PR percentage cutoff to predict outcome being found to be 20\% (Appendix Fig A2-A3, online only). In contrast, within IHC-luminal B/HER2-negative tumors (ie, HR-positive/Ki-67 > 14\%), semiquantitative expression of either PR or ER was not found to be associated with outcome differences (data not shown).

We then tested the prognostic value of the PR cutoff of more than $20 \%$ within IHC-luminal A tumors in two independent cohorts of patients with primary breast cancer (GEICAM $9906^{11}$ and the BCCA-no AST cohorts ${ }^{9}$ ). In both data sets, patients with IHC-luminal A tumors having low positive PR-positive tumor cells $(\leq 20 \%)$ showed significantly poorer survival compared with tumors with more than $20 \%$ of PR-positive tumor cells (Figs $2 \mathrm{~A}$ and $2 \mathrm{~B}$ ). Multivariable analyses confirmed the independent association between PR expression and survival (Appendix Table A4-A5, online

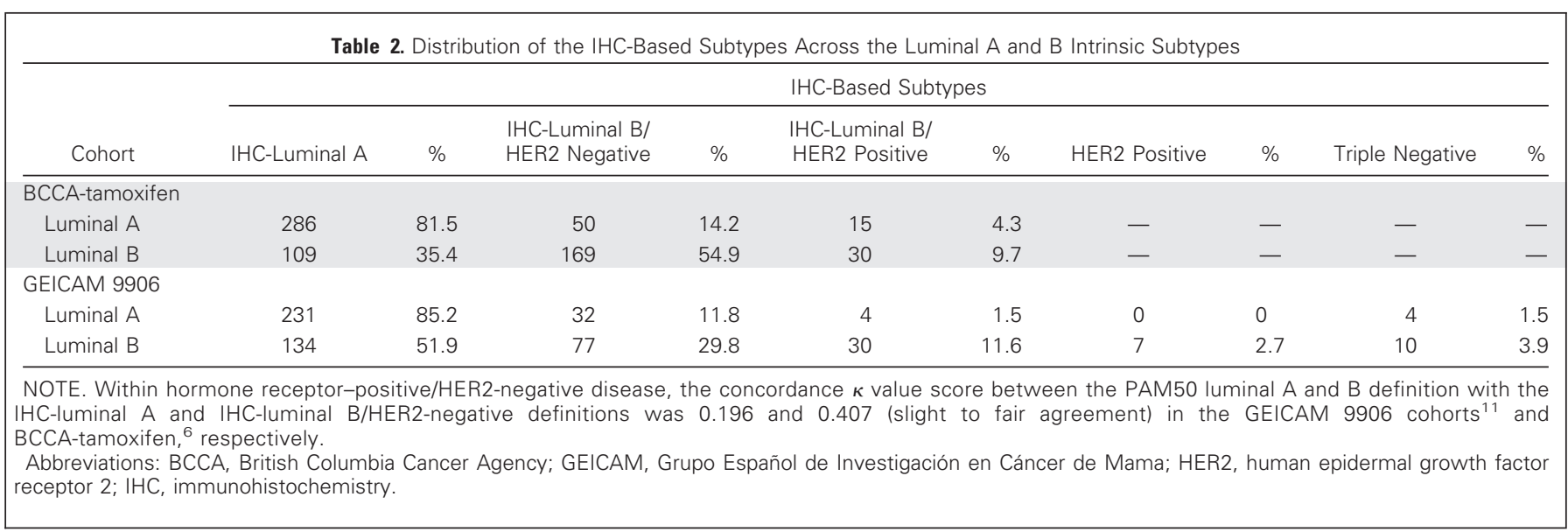



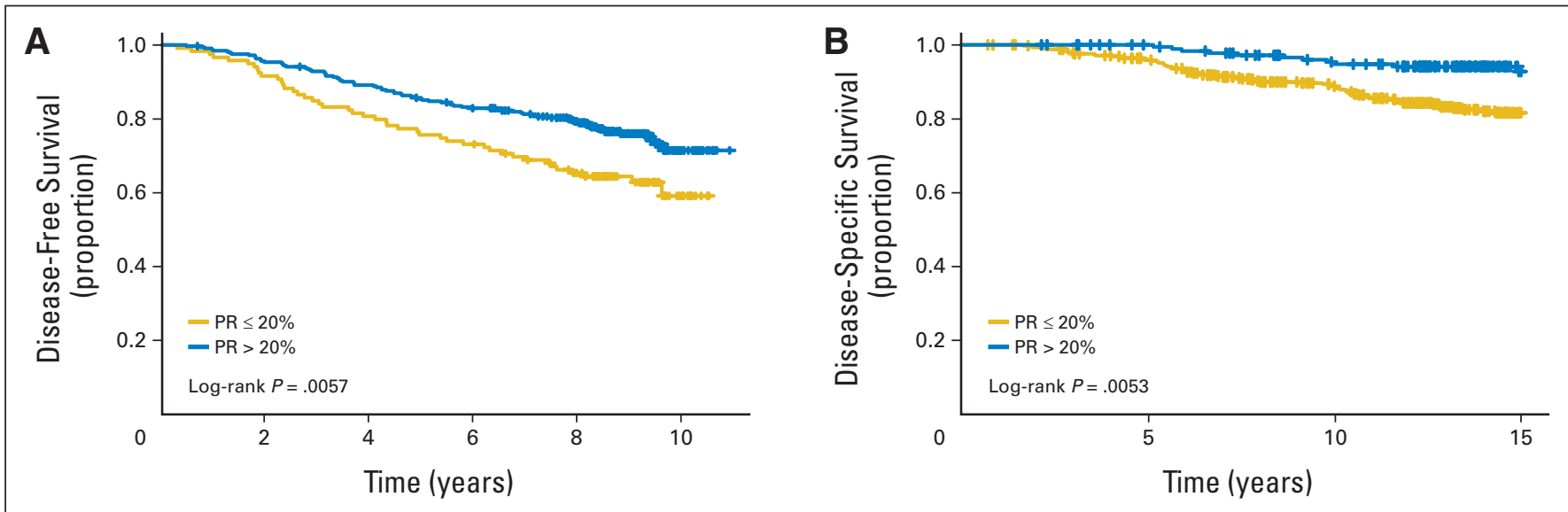

Fig 2. Kaplan-Meier survival analysis within immunohistochemical-based luminal A tumors (hormone receptor positive/HER2 negative/Ki-67 < $14 \%$ ) based on the

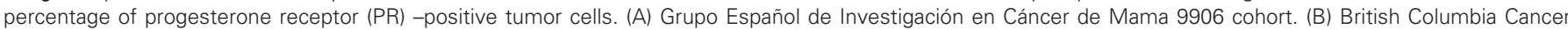
Agency-no adjuvant systemic therapy cohort.

only). In the BCCA-no AST cohort, the breast cancer-specific survival at 15 years of patients with IHC-luminal A tumors with more than 20\% PR-positive tumor cells was $94.0 \%$ (95\% CI, 91.6\% to $98.2 \%$ ).

We next evaluated the distribution of the gene-expression based intrinsic subtypes (gold standard) within IHC-luminal A tumors in the GEICAM 9906 cohort based on this more than 20\% PR cutoff (Table 3). Consistent with the preceding findings, 63\% of IHC-luminal A tumors with more than $20 \%$ of PR-positive cells were identified as luminal A, whereas $24 \%$ of IHC-luminal A tumors with $\leq 20 \%$ of PR-positive cells were identified as luminal A, thus confirming that this definition helps to better discriminate true luminal A tumors from the rest. Finally, although the PR cutoff of $20 \%$ increased the percentage of luminal A tumors identified within what would otherwise have been considered IHCluminal B/HER2-negative tumors from $5.9 \%$ to $30.9 \%$, the majority of this group remained composed of luminal B (55.6\%) tumors.

\section{Comparison of Prognostic Values of IHC-Based Subtypes, IHC4 Score, and PAM50-ROR-P Score}

We compared the contribution of the newly proposed IHCbased subtype definitions (IHC-luminal A [HR positive/HER2 negative/Ki-67 $<14 \% / \mathrm{PR}>20 \%$ ] and IHC-luminal B [HR positive/ HER2 negative/Ki-67 $<14 \% / \mathrm{PR} \leq 20 \%$ or $\mathrm{HR}$ positive/HER2
negative/Ki-67 > 14\%]) with a version of the IHC4 score $^{8}$ and with PAM50 ROR-P score ${ }^{6}$ in the subset of patients with HR-positive/ HER2-negative tumors from the GEICAM 9906 cohort $^{11}(\mathrm{n}=580)$. All three classifications added significant prognostic information beyond clinical variables (Figs 3A, 3B, and 3C), with IHC-based subtypes and IHC4 score providing similar amounts of prognostic information and PAM50 ROR-P providing the largest amount.

Finally, we evaluated the independent prognostic information that each classification provided when considered in the presence of one of the others. When the IHC4 score was included in the model, adding intrinsic IHC-based subtype did not provide significant independent information (Fig 3D). However, when the IHC-based subtype was included in the model, the IHC4 score did not provide additional information (Fig 3E). On the other hand, inclusion of PAM50 ROR-P provided significant independent prognostic information beyond the information provided by either the IHC4 score or the IHC-based subtypes (Figs 3D and 3E).

\section{DISCUSSION}

Patients with early breast cancer with tumors that are ER positive and/or PR positive (ie, luminal) have lower risks of recurrence and

\begin{tabular}{|c|c|c|c|c|c|c|c|c|c|c|c|}
\hline Subtype and PR Status & Luminal A & $\%$ & Luminal B & $\%$ & HER2 Enriched & $\%$ & Basal-Like & $\%$ & Normal-Like & $\%$ & Total \\
\hline IHC-luminal A & 231 & 52.3 & 134 & 30.3 & 56 & 12.7 & 3 & 0.7 & 18 & 4.1 & 442 \\
\hline$P R \leq 20 \%$ & 27 & 22.7 & 61 & 51.3 & 24 & 20.2 & 3 & 2.5 & 4 & 3.4 & 119 \\
\hline$P R>20 \%$ & 204 & 63.2 & 73 & 22.6 & 32 & 9.9 & 0 & 0 & 14 & 4.3 & 323 \\
\hline IHC-luminal B/HER2 negative & 28 & 21.2 & 77 & 58.3 & 19 & 14.4 & 8 & 6.1 & 0 & 0.0 & 132 \\
\hline IHC-luminal B/HER2 positive & 4 & 5.6 & 30 & 41.7 & 38 & 52.8 & 0 & 0.0 & 0 & 0.0 & 72 \\
\hline$P R \leq 20 \%$ & 0 & 0.0 & 13 & 33.3 & 26 & 66.7 & 0 & 0.0 & 0 & 0.0 & 39 \\
\hline$P R>20 \%$ & 4 & 12.1 & 17 & 51.5 & 12 & 36.4 & 0 & 0.0 & 0 & 0.0 & 33 \\
\hline
\end{tabular}

Abbreviations: GEICAM, Grupo Español de Investigación en Cáncer de Mama; HER2, human epidermal growth factor receptor 2; IHC, immunohistochemistry; PR, progesterone receptor. 


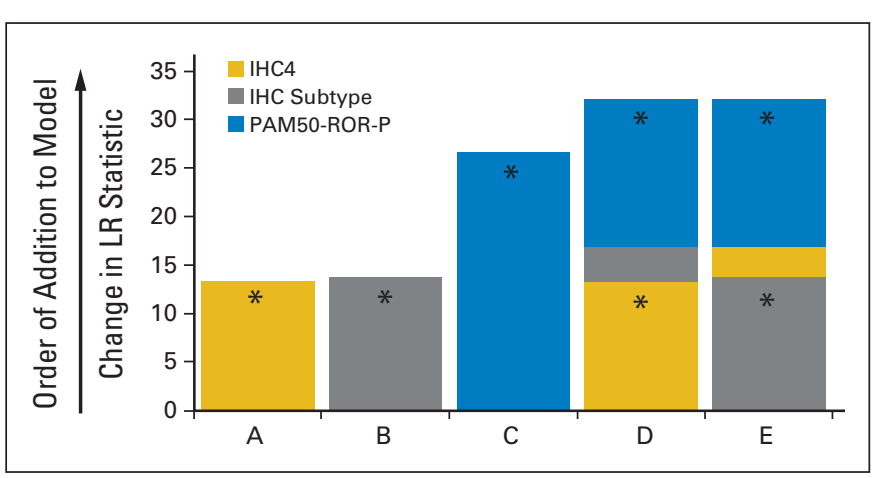

Fig 3. Disease-free survival log likelihood ratio (LR) statistics of six different predictive models (A-E) in patients of the Grupo Español de Investigación en Cáncer de Mama 9906 cohort with hormone receptor (HR) -positive/human epidermal growth factor receptor 2 (HER2) -negative breast cancer. The variables evaluated were the following: immunohistochemical (IHC)-based scoring of estrogen receptor, progesterone receptor, HER2, and Ki-67 (IHC4 score; continuous variable), IHC-based subtypes (HR positive/HER2 negative/Ki-67 $<14 \%$ $>20 \%$ [luminal A], HR positive/HER2 negative/Ki-67 $<14 \% \leq 20 \%$ and HR positive/HER2 negative/Ki-67 > 14\% [luminal B]), and PAM50 risk of recurrence score based on subtype and proliferation (ROR-P; continuous variable). (*) $P<.05$.

mortality compared with women with ER-negative and/or PRnegative disease. ${ }^{3,15}$ However, few studies have evaluated variations in these risks across ER/PR status. ${ }^{15-18}$ In Dunnwald et al, ${ }^{16}$ women with ER-positive/PR-negative, ER-negative/PR-positive, or ER-negative/ PR-negative tumors experienced higher risks of mortality compared with women with ER-positive/PR-positive tumors, independent of the various demographic and clinical tumor characteristics. These data are concordant with our centrally reviewed pathology data presented here, which show that PR positivity, and especially high expression of PR protein, is more frequently observed in tumors with a better baseline prognosis (ie, luminal A) than tumors with a poor baseline prognosis (ie, luminal B). It is important to note that a substantial number of luminal B tumors ( $\sim 50 \%$ to $75 \%$ ) are still PR positive, although the expression of PR may be less than in luminal A tumors.

The ability of ER and/or PR expression to predict benefit to endocrine and/or cytotoxic therapy has also been evaluated. In terms of endocrine sensitivity, a recent patient-level meta-analysis of randomized trials from the Early Breast Cancer Trialists' Collaborative Group that evaluated adjuvant tamoxifen versus no adjuvant tamoxifen suggested that recurrence and death rate ratio is independent of PR status (or level) in ER-positive disease. ${ }^{19}$ Similar data have been observed in another smaller randomized adjuvant study. ${ }^{20}$ In addition, PR expression levels have not shown to predict aromatase inhibitor efficacy over tamoxifen in ER-expressing tumors in two large adjuvant clinical trials. ${ }^{21,22}$ This is concordant with a recent neoadjuvant trial in which luminal A and B tumors, as defined by the PAM50 assay, did not show significant differences in terms of response to aromatase inhibitors, although luminal A tumors achieved higher rates of Preoperative Endocrine Prognostic Index score of 0 , which is a validated biomarker of outstanding outcome after adjuvant endocrine therapy alone. ${ }^{23}$ Overall, these data suggest that luminal A and B tumors benefit similarly from endocrine therapies, but that patients with luminal A tumors have a better baseline prognosis than those with luminal B tumors.

In terms of chemotherapy benefit, the majority of adjuvant and neoadjuvant data suggest that HR status is a strong predictor of general chemosensitivity, with HR-positive tumors showing less benefit to cytotoxic drugs than HR-negative tumors. Moreover, in the neoadjuvant setting, luminal A tumors achieve lower rates of pathologic complete response with anthracycline/taxane-based chemotherapy compared with luminal B tumors. ${ }^{24}$ In addition, Oncotype DX has shown that within HR-positive disease, those tumors with high RS (ie, non-luminal A tumors) benefit the most from adjuvant chemotherapy. ${ }^{25,26}$ Interestingly, in a retrospective analysis from three adjuvant clinical trials, low expression of both ER and PR, and potentially low expression of PR within ER-positive patients, was found predictive of adding chemotherapy to endocrine therapy. ${ }^{27}$ Overall, these data suggest that luminal A tumors are less chemosensitive than luminal Bs.

A critical issue in HR-positive disease is the identification of patients who can be considered virtually cured with endocrine therapy alone and so do not need adjuvant systemic chemotherapy. ${ }^{4,6}$ Gene expression-based assays such as the PAM50 ROR and Oncotype DX RS can help identify these groups of patients, especially within nodenegative disease. ${ }^{28}$ Recently, a combined semiquantitative IHC-based scoring of ER, PR, HER2, and Ki-67, known as IHC4 score, has shown to provide similar prognostic information as is provided by Oncotype DX RS. ${ }^{8}$ In this report, we have shown that a version of the IHC4 score is significantly associated with outcome, but did not add significant prognostic information once our newly improved intrinsic IHCbased subtypes were known within HR-positive/HER2-negative disease. This is probably due to the fact that both pathology-based determinations are using the same four biomarkers to identify similar prognostic groups.

There are several issues that need to be considered in this study. First, the information provided by IHC-based biomarkers cannot simply be used to substitute the information coming from multigenebased assays, and even in the presence of IHC-based assays, the gene expression ROR assay was a strong prognostic feature. However, as stated previously, multigene expression-based assays are not globally available, and in their absence, well-designed IHC assays are valuable for baseline prognostic estimations. A second issue is that many genes were found differentially expressed when luminal A tumors were compared with luminal B tumors, and the quantitative IHC expression of some of these biomarkers could have potentially performed better than PR. However, we decided to focus on the expression of PR because this biomarker is widely used in the community and is already part of the standard assessment at most institutions. Third, the IHCbased subtype definitions evaluated here were performed in a centralized laboratory under a single protocol, and one antibody per protein/ target, which may not reflect the everyday performance of these tests in the clinical setting, where multiple laboratories with different antibodies is more likely to be the approach. Fourth, the IHC4 score evaluated in our study is slightly different from that of Cuzik et $\mathrm{al}^{8}$ as a result of the use of different antibodies for ER and PR and the use of a general intensity score of ER-positive tumor cells. Nonetheless, the association of the IHC4 score with survival was found to be strong, as previously reported. $^{8}$

To conclude, IHC subtype-based definitions of genomically defined luminal $A$ and $B$ tumors are imperfect because of the nature and limitations of pathology-based tests. However, semiquantitative measurement of the percentage of PR-positive cells within HR-positive/ HER2-negative/Ki-67 less than 14\% tumors helps to identify patients who may be considered most effectively treated with endocrine therapy alone. Therefore, the new proposed IHC-based definition of 
luminal A tumors is HR-positive/HER2-negative/Ki-67 less than 14\% and PR more than $20 \%$.

\section{AUTHORS' DISCLOSURES OF POTENTIAL CONFLICTS} OF INTEREST

Although all authors completed the disclosure declaration, the following author(s) and/or an author's immediate family member(s) indicated a financial or other interest that is relevant to the subject matter under consideration in this article. Certain relationships marked with a " $U$ " are those for which no compensation was received; those relationships marked with a " $C$ " were compensated. For a detailed description of the disclosure categories, or for more information about ASCO's conflict of interest policy, please refer to the Author Disclosure Declaration and the Disclosures of Potential Conflicts of Interest section in Information for Contributors. Employment or Leadership Position: None Consultant or Advisory Role: Torsten O. Nielsen, BioClassifier (C) Stock Ownership: Philip S. Bernard, University Genomics, BioClassifier; Charles M. Perou,
University Genomics, BioClassifier Honoraria: None Research Funding: None Expert Testimony: None Other Remuneration: None

\section{AUTHOR CONTRIBUTIONS}

Conception and design: Aleix Prat, Charles M. Perou

Administrative support: Rosalía Caballero

Provision of study materials or patients: Maggie Chon U. Cheang, Miguel Martín, Eva Carrasco, Rosalía Caballero, Philip S. Bernard, Torsten O. Nielsen, Charles M. Perou

Collection and assembly of data: Aleix Prat, Maggie Chon U. Cheang, Miguel Martín, Eva Carrasco, Rosalía Caballero, Philip S. Bernard, Charles M. Perou

Data analysis and interpretation: Aleix Prat, Maggie Chon U. Cheang, Miguel Martín, Joel S. Parker, Scott Tyldesley, Karen Gelmon, Philip S. Bernard, Torsten O. Nielsen, Charles M. Perou

Manuscript writing: All authors

Final approval of manuscript: All authors

\section{REFERENCES}

1. Prat $A$, Perou CM: Deconstructing the molecular portraits of breast cancer. Mol Oncol 5:5-23, 2011

2. Oh DS, Troester MA, Usary J, et al: Estrogenregulated genes predict survival in hormone receptorpositive breast cancers. J Clin Oncol 24:1656-1664, 2006

3. Perou CM, Sørlie T, Eisen MB, et al: Molecular portraits of human breast tumours. Nature 406: 747-752, 2000

4. Prat A, Ellis MJ, Perou CM: Practical implications of gene-expression-based assays for breast oncologists. Nat Rev Clin Oncol 9:48-57, 2012

5. Parker JS, Mullins $M$, Cheang $M C$, et al: Supervised risk predictor of breast cancer based on intrinsic subtypes. J Clin Oncol 27:1160-1167, 2009

6. Nielsen TO, Parker JS, Leung $S$, et al: A comparison of PAM50 intrinsic subtyping with immunohistochemistry and clinical prognostic factors in tamoxifen-treated estrogen receptor-positive breast cancer. Clin Cancer Res 16:5222-5232, 2010

7. Allison $\mathrm{KH}$, Kandalaft $\mathrm{PL}$, Sitlani $\mathrm{CM}$, et al: Routine pathologic parameters can predict Oncotype DX(TM) recurrence scores in subsets of ER positive patients: Who does not always need testing? Breast Cancer Res Treat 131:413-424, 2012

8. Cuzick J, Dowsett M, Pineda S, et al: Prognostic value of a combined estrogen receptor, progesterone receptor, Ki-67, and human epidermal growth factor receptor 2 immunohistochemical score and comparison with the Genomic Health recurrence score in early breast cancer. J Clin Oncol 29:4273-4278, 2011

9. Cheang MC, Chia SK, Voduc D, et al: Ki67 index, HER2 status, and prognosis of patients with luminal B breast cancer. J Natl Cancer Inst 101:736750, 2009

10. Goldhirsch A, Wood WC, Coates AS, et al: Strategies for subtypes-dealing with the diversity of breast cancer: Highlights of the St. Gallen International Expert Consensus on the Primary Therapy of Early Breast Cancer 2011. Ann Oncol 22:1736-1747, 2011
11. Martín M, Rodríguez-Lescure A, Ruiz A, et al: Randomized phase 3 trial of fluorouracil, epirubicin, and cyclophosphamide alone or followed by paclitaxel for early breast cancer. J Natl Cancer Inst 100:805-814, 2008

12. Martín M, Rodríguez-Lescure A, Ruiz A, et al: Molecular predictors of efficacy of adjuvant weekly paclitaxel in early breast cancer. Breast Cancer Res Treat 123:149-157, 2010

13. Genetic Pathology Evaluation Centre: TMA viewer. http://www.gpecimage.ubc.ca/

14. Huang DW, Sherman BT, Lempicki RA: Systematic and integrative analysis of large gene lists using DAVID bioinformatics resources. Nat Protocols 4:44-57, 2009

15. Bauer K, Parise C, Caggiano V: Use of ER/PR/ HER2 subtypes in conjunction with the 2007 St Gallen Consensus Statement for early breast cancer. BMC Cancer 10:228, 2010

16. Dunnwald L, Rossing M, Li C: Hormone receptor status, tumor characteristics, and prognosis: a prospective cohort of breast cancer patients. Breast Cancer Res 9:R6, 2007

17. Grann VR, Troxel AB, Zojwalla NJ, et al: Hormone receptor status and survival in a populationbased cohort of patients with breast carcinoma. Cancer 103:2241-2251, 2005

18. Mohsin SK, Weiss $H$, Havighurst $T$, et al: Progesterone receptor by immunohistochemistry and clinical outcome in breast cancer: A validation study. Mod Pathol 17:1545-1554, 2004

19. Early Breast Cancer Trialists' Collaborative Group (EGCTCG), Davies C, Godwin J, et al: Relevance of breast cancer hormone receptors and other factors to the efficacy of adjuvant tamoxifen: Patient-level meta-analysis of randomised trials. Lancet 378:771-784, 2011

20. Dowsett M, Houghton J, Iden C, et al: Benefit from adjuvant tamoxifen therapy in primary breast cancer patients according oestrogen receptor, progesterone receptor, EGF receptor and HER2 status. Ann Oncol 17:818-826, 2006

21. Viale G, Regan MM, Maiorano E, et al: Prognostic and predictive value of centrally reviewed expres- sion of estrogen and progesterone receptors in a randomized trial comparing letrozole and tamoxifen adjuvant therapy for postmenopausal early breast cancer: BIG 1-98. J Clin Oncol 25:3846-3852, 2007

22. Dowsett M, Allred C, Knox J, et al: Relationship between quantitative estrogen and progesterone receptor expression and human epidermal growth factor receptor 2 (HER-2) status with recurrence in the Arimidex, Tamoxifen, Alone or in Combination Trial. J Clin Oncol 26:1059-1065, 2008

23. Ellis MJ, Suman VJ, Hoog J, et al: ACOSOG Z1031, a randomized phase 2 neoadjuvant comparison between letrozole, anastrozole and exemestane for postmenopausal women with ER rich stage 2/3 breast cancer: Clinical and biomarker outcomes and the predictive value of the baseline PAM50based intrinsic subtype-ACOSOG Z1031. J Clin Oncol 29:2342-2349, 2011

24. Parker J, Prat $A$, Cheang $M$, et al: Breast cancer molecular subtypes predict response to anthracycline/ taxane-based chemotherapy. Presented at the San Antonio Breast Cancer Symposium, San Antonio, TX, December 9-13, 2009 (abstr 2019)

25. Albain KS, Barlow WE, Shak S, et al: Prognostic and predictive value of the 21-gene recurrence score assay in postmenopausal women with nodepositive, oestrogen-receptor-positive breast cancer on chemotherapy: A retrospective analysis of a randomised trial. Lancet Oncol 11:55-65, 2010

26. Paik S, Tang G, Shak S, et al: Gene expression and benefit of chemotherapy in women with node negative, estrogen receptor positive breast cancer. J Clin Oncol 24:3726-3734, 2006

27. Viale G, Regan MM, Maiorano E, et al: Chemoendocrine compared with endocrine adjuvant therapies for node-negative breast cancer: Predictive value of centrally reviewed expression of estrogen and progesterone receptors-An International Breast Cancer Study Group. J Clin Oncol 26:1404-1410, 2008

28. Prat A, Parker J, Fan C, et al: Concordance among gene-expression-based predictors for ERpositive breast cancer treated with adjuvant tamoxifen. Ann Oncol lepub ahead of print on April 24, 2012] 